\title{
Faire de loisirs travail : les conditions sociales des reconversions professionnelles dans le tourisme
}

\section{Tristan Loloum}

To cite this article: Tristan Loloum (2018) Faire de loisirs travail : les conditions sociales des reconversions professionnelles dans le tourisme, Loisir et Société / Society and Leisure, 41:2, 311-330, DOI: 10.1080/07053436.2018.1482677

To link to this article: https://doi.org/10.1080/07053436.2018.1482677

冓 Published online: 20 Jul 2018.

Submit your article to this journal $\sqrt{6}$

Џll Article views: 6

View Crossmark data $[\pi$ 


\title{
Faire de loisirs travail : les conditions sociales des reconversions professionnelles dans le tourisme
}

\author{
Tristan Loloum* \\ Université de Lausanne, FGSE, Sion, Suisse \\ (Received 17 November 2016; accepted 31 May 2017)
}

\begin{abstract}
To what extent do leisure and travel participate in creating tourism professionals? How does one undertake a professional transition toward travel and tourism? Turning travel experiences into a job or leisure into a professional vocation is an increasingly widespread ambition. In the media, it is common to find incentives inviting anyone to leave his/her job and 'earn money while traveling around the world.' Against such commonsense discourses that tend to present passion, courage, and individual talent as the key elements of successful professional transition toward tourism and leisure jobs, the sociological analysis insists on the objective limitations and specific resources that are necessary to turn a passion into work, a travel experience into a livelihood, and a lifestyle into a commercial enterprise. Therefore, making a living out of travel and leisure is like 'making a virtue out of a need': it is pretending - advantageously - that a trajectory predetermined by social dispositions is the result of personal willingness and hedonistic motivations.
\end{abstract}

Keywords: tourism; lifestyle; international capital; biographical change

En quoi les loisirs et les voyages participent-ils à forger des professionnels du tourisme? Comment est-ce qu'on entreprend une reconversion dans les métiers du tourisme et de l'hôtellerie? Faire du voyage un travail ou de ses loisirs un métier est une ambition contemporaine de plus en plus répandue. Contre les injonctions au voyage invitant à tout quitter pour « gagner sa vie en voyageant», et posant la passion, le courage et le talent individuel comme uniques moteurs des reconversions par les loisirs, l'analyse sociologique des trajectoires d'entrepreneurs touristiques révèle les contraintes objectives et les ressources spécifiques nécessaires à ériger une passion en métier, un voyage en gagne-pain et un style de vie en entreprise commerciale. Dès lors, faire de ses voyages et loisirs un travail, c'est comme « faire de nécessité vertu » : c'est faire passer - à bon compte - une trajectoire prédéterminée par des dispositions sociales pour le produit d'une volonté personnelle et d'une attitude hédoniste.

Mots clés : tourisme; style de vie; capital international; reconversion

Plus radical encore que de quitter son travail pour voyager (à la faveur d'un congé sabbatique ou d'une démission fracassante), faire des voyages un métier est une ambition contemporaine de plus en plus répandue. Dans les médias, les "injonctions au voyage » (Réau, 2011) et les bons conseils ne manquent pas pour inviter tout un chacun à « tout quitter pour gagner sa vie en voyageant », que ce soit pour aller cueillir des fraises en Nouvelle-Zélande, créer son propre «travel blog » ou sa chaîne YouTube. Les magazines de compagnies aériennes low-cost font les louanges d'une vie hypermobile, confondant voyages d'affaires et tourisme, expatriation et voyages. On y décrit à longueur de page le 
quotidien d'un hypothétique « get-up-and-go generation ${ }^{1} »$, de « remote workers » et autres « roamers » (Patha, 2016) - ces jeunes cadres vagabonds se déplaçant de pays en pays à la poursuite d'objectifs personnels et professionnels. L'émergence des « tiers-lieux ${ }^{2}$ », proposant de concilier plaisirs de voyage et astreintes professionnelles, s'inscrit également dans cette tendance supposément générale à l'hybridation des temps de travail et des lieux de loisirs.

Difficile pourtant de ne pas se montrer perplexe face à une telle banalisation du voyage, qui interroge sur la distribution sociologique de la mobilité professionnelle (Viry, Kaufmann, et Widmer, 2009), sur les coûts de l'expatriation, souvent déguisée en choix de vie même lorsqu'elle est contrainte (Guillaume et Pochic, 2010), ou sur la précarité que dissimule l'octroi de visas «vacances-travail » à de jeunes travailleurs et autres "woofers » en quête d'expériences internationales à moindres frais (Perrier, 2015, pp. 36-38). Il est bon en effet de rappeler que loisirs et hypermobilité n'échappent pas à certains déterminismes sociologiques et à certains coûts financiers. ${ }^{3}$

L'hybridation des temps et des lieux associés au travail et au loisir n'est pas de fait une nouveauté pour les milieux favorisés. La « classe des loisirs » dont parlait Veblen (1899) n'est pas simplement « oisive » : elle a toujours activement travaillé à faire des loisirs une source de légitimité. Le sport, les voyages, l'art sont en effet des « activités qui témoignent de prouesses [des élites] en mettant à profit leur tempérament (promptitude à s'affirmer, à rivaliser, à prendre l'offensive) et leur physique (plus vigoureux et massif, plus apte à des tensions soudaines et violentes) » (Lafortune, 2007).

Sous quelles conditions dès lors peut-on faire de ses voyages et de ses loisirs un gagne-pain? Comment la mobilité et les loisirs peuvent-ils être mis à contribution dans les trajectoires professionnelles? L'hypothèse explorée dans ce travail est que l'expérience acquise dans les voyages et les loisirs peut être convertie en ressources professionnelles/ commerciales à deux conditions : d'une part, il est préférable qu'il s'agisse de loisirs socialement et culturellement valorisés, en accord avec une demande existante ou émergente; d'autre part, il faut que cette expérience des voyages et des loisirs soit combinée avec d'autres types de ressources, comme un capital foncier ou financier, un réseau de connaissances, des savoir-faire professionnels ou des savoir-vivre fongibles dans le nouveau métier. L'enquête suggère également que ces reconversions ont lieu bien souvent à des moments particuliers du cycle de vie, et lorsque les schémas traditionnels de réussite professionnelle sont moins attractifs ou moins accessibles.

\section{Au-delà des illusions hérö̈ques : profits symboliques des loisirs et conditions sociales des reconversions dans le tourisme}

Être un «bon voyageur » (aventureux, débrouillard, communicatif, innovant) ne s'apprend pas uniquement dans le voyage, mais à travers l'acquisition de compétences relationnelles, linguistiques et culturelles qui s'acquièrent en amont de la mobilité géographique. Il ne suffit pas d'avoir soi-même beaucoup voyagé pour réussir dans un emploi à vocation internationale ou touristique. L'acquisition et la rentabilité d'un « capital international » dépendent toujours d'autres formes de capitaux acquis en dehors de l'expérience de voyage (Wagner, 2007). Pour saisir l'impact de l'internationalisation sur les trajectoires professionnelles, il faut dès lors considérer les chances de mobilité au regard de l'origine sociale, des configurations familiales et des étapes du cycle de vie dans lesquels se trouvent les individus, ainsi que les particularités du domaine d'activité concerné (ici, le tourisme). 
À l'encontre des discours posant la passion, le courage et le talent individuel comme uniques moteurs des reconversions par les loisirs, l'analyse sociologique invite à réfléchir aux contraintes objectives et aux ressources spécifiques nécessaires à ériger une passion en métier, un voyage en gagne-pain et un style de vie en entreprise commerciale. De nombreux travaux ont déjà étudié le reclassement des élites à travers le tourisme et les loisirs (Bertho Lavenir, 1999; Réau, 2011; Saint Martin, 2000). Les mérites de ces enquêtes sociologiques sont de rompre avec l'illusion des inventions ex nihilo; de mettre en doute certaines idées reçues annonçant l'avènement d'une " civilisation des loisirs » (Dumazedier, 1962) qui serait caractérisée par une dissolution progressive des frontières entre loisirs, voyages et travail; de replacer ces « ruptures » dans la continuité d'un espace de possibles en les interprétant au regard des dispositions objectives et des contraintes collectives qui pèsent sur les individus. Dès lors, faire de ses voyages et loisirs un travail, c'est comme « faire de nécessité vertu » : c'est faire passer - à bon compte - une trajectoire prédéterminée par des dispositions sociales pour le produit d'une volonté personnelle et d'une attitude hédoniste ou subversive.

Cette étude s'inscrit dans une réflexion sur les trajectoires de reconversion professionnelle. Elle vise à remettre en cause « l'illusion héroïque » qui tend à faire des ruptures professionnelles des «moments du règne des facteurs subjectifs » (Dobry, 2010, p. 67). Les reconversions dans les métiers du tourisme sont en effet souvent présentées comme des « bifurcations » (Grossetti, 2010), c'est-à-dire comme des changements radicaux et irréversibles où la nouvelle séquence dans le parcours de vie serait sans lien avec la précédente. Il est vrai que le choix de s'installer comme hôtelier dans une petite station balnéaire après avoir exercé dans la fonction publique apparaît a priori comme un « tournant majeur » (Abbott, 2001). L'hypothèse de « transposabilité des dispositions » permet cependant de saisir les critères de continuité dans ces parcours apparemment contingents. Cette hypothèse, au cœur de la notion " d'habitus ${ }^{4}$ " (Bourdieu, 1980), suppose que des dispositions acquises dans une certaine activité sociale (par exemple la famille ou les voyages) puissent être transposées dans une autre (par exemple les métiers $\mathrm{du}$ tourisme) sans qu'il s'agisse d'une opération consciente. Il ne s'agit donc pas de bifurcation, au sens où l'on ferait table rase du passé, mais de «conversion » de différentes espèces de capitaux (Bourdieu, 1979, p. 137) dans une nouvelle activité.

Partant d'entretiens biographiques avec des entrepreneurs touristiques d'une station balnéaire du Nordeste brésilien (cf. encadré), cet article cherche à expliquer comment des surfeurs et des voyageurs adeptes de la contre-culture hippie dans les années 1970 ont pu devenir des fers de lance du développement économique local. On interroge le rôle du surf et des voyages dans leurs parcours professionnels, éléments présentés par les intéressés comme des « détonateurs » par bon nombre d'enquêtés. En quoi leur expérience de l'international, des nouveaux loisirs comme le surf et de la contre-culture des années 1970 a-t-elle aidé à forger leurs trajectoires professionnelles? Et surtout, quels autres types de ressources objectives l'évocation nostalgique de ces activités distinctives dissimule-t-elle? Il apparaît que le surf et les voyages sont des paravents légitimateurs à un processus de reconversion sociologique autrement plus complexe. Issus de milieux favorisés, ces individus ont surtout su convertir opportunément des ressources économiques, sociales et culturelles en une offre touristique nouvelle, alors même que les débouchés « ordinaires » auxquels leurs milieux d'origine les prédestinaient apparaissaient limités.

Lorsqu'on interroge les hôteliers historiques de Pipa, l'appartenance - de près ou de loin - au monde du surf revient presque systématiquement dans la description de leur trajectoire, comme si le souvenir d'un passé baigné par l'insouciance d'une vie sans attache conférait un gage d'authenticité à leur situation actuelle. Ce recours quasi magique 
à l'univers du surf comme argument de légitimation évoque les travaux du sociologue Christophe Guibert dans le sud-ouest de la France sur l'usage homogénéisant et opportuniste de la catégorie "surfeur », qui tend à gommer l'origine sociale favorisée des premiers pratiquants, tout en leur attribuant les profits symboliques d'un style de vie associé à la « contre-culture », au voyage, à la nature et à l'ascétisme : « Activité gratuite et désintéressée, le surf est pour ces individus un moyen d'entretenir et de diversifier un capital social et symbolique déjà important »(Guibert, 2007, p. 95).

Au-delà de sa rentabilité symbolique, Guibert montre que « l'esprit surf » est aussi une recette commerciale particulièrement vendeuse, aussi bien pour les magazines spécialisés (principaux relayeurs en France du mythe du surfeur rebelle nord-américain héros de la Beat Generation et de la culture hippie) que pour les marques de surfwear et autres entreprises intéressées de près ou de loin au négoce du surf, à l'image de ces promoteurs du surf interviewés par le sociologue : «Toute une génération de gens a envie de rester scotchée sur les années 1970. Parce que c'est vendeur! » (Guibert, 2011, p. 33). Ou encore : « Ceux qui achètent nos produits doivent garder ce sentiment d'appartenance, cette dimension d'évasion, de rêve, de rencontre avec l'histoire du surf » (p. 39).

Il en va du surf comme des voyages, et nombre d'entrepreneurs mettent en avant leur capital international - leur expérience des voyages, leur maitrise des langues ou des codes culturels d'autres pays - pour expliquer leurs prédispositions aux métiers du tourisme :

Cette culture internationale est à la source de profits sociaux et professionnels, mais aussi de rétributions symboliques non négligeables. Celui qui peut mener sa vie à l'échelle internationale revendique la supériorité de son point de vue sur celui qui est cantonné au « local ». Le voyage dote de qualités pensées en termes moraux : il atteste de l'ouverture d'esprit, de la curiosité et d'un certain goût du risque. En ce sens, le voyage forme aussi l'entrepreneur, celui qui se définit par sa capacité à innover et à s'affranchir des routines. Certains récits de voyage dans les plus hautes classes donnent bien à voir cet usage légitimateur. (Wagner, 2007, p. 60)

On peut se demander dès lors en quoi et sous quelles conditions l'héritage du surf et l'expérience des voyages ont pu aider des individus à faire recette dans le tourisme. Comment sont-ils passés de surfeurs et voyageurs à entrepreneurs hôteliers ou restaurateurs?

\section{Méthodologie}

\section{Entretiens biographiques}

L'enquête repose essentiellement sur une dizaine d'entretiens biographiques avec des entrepreneurs pionniers de Praia da Pipa fréquentant la station depuis les années 1970-80 et installés en tant que professionnels depuis les 1980-90. En particulier, l'analyse approfondie des trajectoires de six individus (quatre hôteliers, deux restaurateurs) permet de comprendre comment des qualités et des ressources acquises en dehors du diplôme et du travail peuvent expliquer les modalités de conversion aux métiers du tourisme.

L'approche biographique (Bertaux, 1980) permet de reconstituer l'ensemble des capitaux mobilisés par les individus à des moments précis des différents cycles de vie. Au cours des entretiens, nous étions particulièrement attentifs aux moments de rupture, aux «tournants de carrières " (Hughes, 1996), où les recompositions de capitaux étaient susceptibles d'avoir lieu. Pour E. C. Hughes, les trajectoires individuelles sont composées de différents cycles (biologique, institutionnel, familial) se 
déroulant dans des espaces sociaux différents (le couple, les amis, la profession, les loisirs, etc.). La circulation entre ces différents espaces sociaux est organisée autour de moments pivots, ritualisés ou non, au cours desquels les individus peuvent réorienter leur activité en recomposant leur portefeuille d'investissements biographiques (ou capitaux). Reconstituer ces parcours permet en outre de comprendre les règles de circulation dans l'espace social et illustrer la structure d'un champ, en l'occurrence ici, la structure du champ touristique dans une station émergente brésilienne.

\section{Le lieu d'enquête}

Ces éléments biographiques s'ancrent à leur tour dans l'ethnographie d'une station balnéaire (Pipa) réalisée dans le cadre d'un travail de thèse entre 2010 et 2014 (Loloum, 2015a). Comme le montre Bertrand Réau (2011) au sujet de l'émergence de nouvelles formules de loisirs en France, l'offre et la demande ne se rencontrent pas abstraitement sur un marché. La sociogenèse de la destination permet ainsi de prendre en compte à la fois les modes de vie, les trajectoires, les goûts culturels de ses promoteurs (l'offre) avec le contexte territorial et les médiations sociales qui rendent possible la convergence de cette offre avec un public réceptif (la demande).

Praia da Pipa est une destination où le surf et la bohème ont fait recette. Dans les étroites rues pavées de la station, d'athlétiques surfeuses et surfeurs défilent - planche sous le bras - entre les touristes, les boutiques de modes, les devantures d'hôtels et les restaurants aux influences cosmopolites : des pizzerias italiennes aux churrascarias argentines en passant par les crêperies bretonnes, les bars à tapas et les sushi-shops, sans oublier les typiques tapiocarías, honorant la gastronomie nordestine en la modernisant. Pipa se veut « hippie chic » et tente tant bien que mal de préserver son aspect de « village » en dépit de l'affluence grandissante des touristes, des promoteurs immobiliers et des festivaliers venant en masse assister aux concerts de plus en plus fréquents de sertanejo ou de forró electrônico promus par l'industrie musicale régionale. En 2015, Pipa comptait environ 6 000 habitants et à peu près autant de lits touristiques, mis à disposition par de petits et moyens établissements : hôtels de charme, pousadas (pensions), bed and breakfast et autres résidences sécurisées en location. Inspiré par les styles de vie du surf et la culture hippie, véritables mythes fondateurs de la station, l'entrepreneuriat hôtelier y cultive le style faussement « rustique » et « décalé » des premiers surfeurs, des « jeunes bien élevés qui faisaient semblant de ne pas l'être » (Jaxel-Truer, 2016). Ce serait eux - les surfeurs qui auraient « découvert » Pipa dans les années 1970 et l'auraient fait connaitre dans le monde entier.

La première partie de l'article insiste sur le rôle des « surfeurs » dans le développement de la station et replace la pratique du surf dans le contexte social et politique brésilien de l'après-guerre. L'analyse sociohistorique de cette catégorie de population révèle les éléments qui ont poussé ces jeunes urbains issus d'un milieu social privilégié à s'installer dans des régions reculées du littoral (l'influence de la culture nord-américaine, la saturation ou dévalorisation des débouchés professionnels traditionnels, la revalorisation stratégique et culturelle du littoral) et leur ont permis d'y démarrer avec succès des entreprises touristiques (la mobilisation de ressources économiques, sociales et familiales, l'usage stratégique de la mobilité, l'adéquation sociologique de l'offre commerciale avec une demande touristique émergente). La deuxième partie s'intéresse à un autre groupe - les descendants de l'élite 
agraire - et étudie comment leurs goûts pour les voyages et les sports d'élite ont été convertis commercialement dans le cadre d'entreprises hôtelières. On montre que ces expériences de l'international et des loisirs n'ont de véritable valeur pour l'entreprise qu'une fois combinées avec d'autres formes de ressources (un capital foncier, social, économique, culturel). Il faut par ailleurs mettre en perspective le choix de reconversion avec certaines contraintes objectives du groupe en question (le déclin des plantations et des schémas traditionnels de réussite professionnelle).

\section{De surfeurs à entrepreneurs : la jeunesse bourgeoise et contre-culturelle comme avant-garde touristique}

Bien que la villégiature à Pipa remonte au début du vingtième siècle, lorsque les maîtres des plantations sucrières alentour venaient s'initier aux premiers bains de mer, c'est véritablement dans les années 1970 et 80 que le tourisme commence à s'y développer sous l'impulsion de jeunes « surfeurs » venus des métropoles voisines (Natal, João Pessoa, Recife) et du sud-est du pays (Rio de Janeiro, São Paulo, Rio Grande do Sul). D'abord présents par intermittence, pour les vacances ou les longs week-ends qu'offre la vie étudiante, nombre d'entre eux vont développer une affinité particulière avec le lieu et ouvrir les premières entreprises touristiques de la station : hôtels, restaurants, agences de tourisme. Pour comprendre le passage de « surfeur » à hôtelier ou restaurateur, il faut non seulement interroger cette catégorie de « surfeur » et la replacer dans les transformations de la société brésilienne dans la deuxième moitié du vingtième siècle, mais aussi comprendre les modalités de conversion de ressources et de professionnalisation dans un secteur d'activité encore peu développé dans cette région.

\section{Le surf comme pratique distinctive}

Les années 1970 et 80 sont propices à l'émergence de nouvelles pratiques de loisirs, nouvelles destinations touristiques et de nouvelles vocations professionnelles parmi la jeunesse favorisée brésilienne. Bien qu'importé des États-Unis pour la première fois dans les années $1930,{ }^{5}$ ce n'est qu'après la Seconde Guerre mondiale que le surf se popularise au Brésil, dans un contexte de profondes transformations économiques et sociales, d'expansion de l'influence nord-américaine et de répression des mouvements contestataires. Initiées sous l'ère Vargas (1930-45), ces transformations se prolongent avec la dictature militaire de 1964-85. L'État amorce à partir de 1930 un nouveau processus d'industrialisation, de centralisation du pouvoir, de réunification territoriale du pays et de refondation de la conscience nationale (Garcia, 1993a). Ces nouvelles dynamiques facilitent les circulations entre les différentes régions et font du littoral un nouvel enjeu stratégique (pour l'industrie, le transport, l'exploitation du pétrole et la défense du territoire). Le rapprochement avec les États-Unis pendant la Seconde Guerre mondiale et l'installation de nombreuses bases militaires américaines sur le littoral brésilien ${ }^{6}$ marquent un changement dans l'influence des modèles étrangers : les normes européennes cèdent le pas aux valeurs américaines.

Le champ sportif exprime en partie ces transformations en « offrant des occasions originales à des groupes particuliers de se manifester sur un mode éthique; en s'opposant, ils tentent de définir ce que doit être la vraie vie, ce qui relève ou non du sport et ce qui fait la valeur des membres associés dans le groupement sportif »(Defrance, 1989, p. 76). Au Brésil, le développement des sports aquatiques est le signe d'une reconquête symbolique d'un espace urbain autrefois relégué : la plage. Dans une société agraire et patriarcale (faisant la part belle aux sports équestres), l'aviron, la voile et la plongée étaient déjà au 
début du siècle - bien avant le surf - une manière pour une partie de la jeune élite urbaine de sortir de l'ombre de leurs aînés et de se distinguer (Lucena, 2000, pp. 120-124).

Capitale touristique internationale, Rio de Janeiro est la porte d'entrée principale pour les surfeurs étrangers qui diffusent de nouvelles techniques et de nouveaux matériaux parmi la jeunesse favorisée carioca (Arias, 2002). La Cidade Maravilhosa connaît son premier groupe de surfeurs dans les années 1950. Les spots de l'Arpoador et de la Jetée d'Ipanema deviennent des lieux cultes du surf brésilien où vont se faire connaître les premiers surfeurs professionnels, fabricants de planches et autres journalistes spécialisés. Dans l'État du Rio Grande do Norte, la première pratique du surf remonte aux années 1960 et est attribuée à deux frères cariocas, Marquinhos et Rosset, fils d'un militaire muté à Natal (Oliveira, 2015). Étudiants dans un riche lycée privé, ils transmettent rapidement leur passion à d'autres jeunes issus des milieux aisés de la ville. Le Nordeste, mis en avant dans la littérature de la première moitié du vingtième siècle qui en fait un bastion de l'authenticité rurale et de la conscience nationale (Garcia, 1993a, p. 32), attire alors beaucoup de jeunes voyageurs du sud-est urbain et industriel.

Socialement distinctif, le surf est interprété par certains auteurs comme Marcos Napolitano (2002) comme une forme non conventionnelle d'expression politique, dans un pays en proie à la répression des mouvements d'oppositions et à la censure des canaux traditionnels de diffusion des idées politiques (livres, places publiques), tant sous l'Estado Novo de Vargas (1937-45) que sous la dictature de Videla. L'art, la musique et la plage deviennent dès lors de nouveaux espaces sociaux de contestation. Exempt de références marxistes, le surf amorce un nouveau style d'opposition culturelle inspiré de la contreculture nord-américaine : des cheveux longs, des tatouages, un jargon particulier, l'usage de drogues, une forme de détachement matériel, etc.

Il faut toutefois nuancer le mythe du surfeur déviant, refusant les règles de vie en société et détaché des contraintes matérielles en soulignant non seulement le caractère « sélectif » de la pratique (Guibert, 2007), mais aussi le conformisme paradoxal des Beach Boys à un projet politique national (la formation d'une conscience nationale et l'unification du territoire par l'exploration des marges), à l'influence culturelle nord-américaine et à des formes policées de subversion. Contre l'idée d'un sport libéré des structures institutionnelles, il faut enfin rappeler l'importance des organisations associatives (qui organisent championnats et compétitions) et du marché dans la diffusion de la pratique (Arias, 2002; Guibert, 2011). La professionnalisation et l'institutionnalisation du surf dans les années 1970-80 s'accompagnent d'une augmentation de la compétition, de l'amélioration des techniques et de l'insertion du surf dans l'espace médiatique; tandis que les années 1990 consacrent la « spectacularisation » au niveau mondial du surf, et par conséquent des hauts-lieux de pratique.

\section{La réinvention touristique du littoral}

Dans le Rio Grande do Norte, les premières compétitions organisées à Natal dans les années 1970 mettent en lumière de jeunes champions qui deviendront des figures du surf au niveau régional et national. Parmi eux se trouvent les surfeurs qui " découvriront » Pipa. L'exploration du littoral à la recherche de nouveaux spots occupe la plupart de leurs week-ends et vacances, certains parvenant même à bénéficier du soutien de commanditaires officiels. Le premier surfeur à se rendre à Pipa est un fils de bonne famille en couple avec une fille issue de l'aristocratie sucrière locale, habituée à y passer ses vacances estivales. Le nom de Praia da Pipa se répand rapidement dans le petit monde du surf brésilien et ses magazines, et même au-delà, parmi les voyageurs de tous horizons arpentant le littoral de station en station. 
L'arrivée de ces nouveaux visiteurs bouscule le quotidien tranquille du village, occupé en majorité par des familles de paysans-pêcheurs, et en été par quelques familles de villégiateurs. Ils sont fils de militaires, de magistrats, de commerçants, de médecins, de professeurs ou de propriétaires terriens. Leur style anticonformiste s'exprime à travers des pratiques sportives (surf, capoeira) et des goûts esthétiques (intérêt pour les arts populaires, mouvements musicaux Bossa Nova, Tropicalía, musique populaire brésilienne) en rupture avec le conservatisme de l'époque (Rivron, 2007). À Pipa, malgré le fossé social et culturel existant entre jeunes bourgeois des villes et paysans pêcheurs du littoral, il se tisse entre eux une affinité bien différente de l'amitié très hiérarchique qui liait les familles autochtones aux premiers villégiateurs (Loloum, 2015b). Aventureux, les jeunes surfeurs se formalisent moins des distinctions de statut et du confort matériel que les estivants traditionnels. Alors que ces derniers débarquaient chargés de bagages et de denrées pour tenir durant tout l'été, les surfeurs eux voyagent légers. À la différence des estivants, ils ne viennent pas avec leurs employés domestiques, mais font appel plutôt à des locaux pour préparer leurs repas quotidiens, à l'image de Dona Eunice qui transforme sa maison en restaurant pour surfeurs. Ils ne viennent pas non plus en famille pendant les grandes vacances, à l'instar des villégiateurs, mais plutôt entre amis, pendant les longs week-ends prolongés qu'offre la vie étudiante. Au début, ils campent indifféremment sous les auvents des villas inhabitées des estivants, dans les jardins des locaux, voire à même la plage. Contrairement aux villégiateurs traditionnels, encore emprunts des modes ruraux de sociabilité, les surfeurs ne partagent pas vis-à-vis des locaux le même sentiment de supériorité que l'aristocratie agraire.

Ces nouveaux visiteurs portent un regard nouveau sur le style de vie des populations du littoral, longtemps ignorés dans l'histoire brésilienne (Dantas, 2009). La « simplicité » des habitants autochtones n'est pas vue comme une marque d'infériorité, mais plutôt « d'authenticité ». La communauté locale devient elle-même un objet d'attraction, et non plus seulement le panorama côtier. C'est en partie cet « art de vivre local » (le jeitinho nativo), anti-modèle de la ville impersonnelle et chaotique, qu'ils sont venus rechercher. Leur intérêt se porte autant sur la plage que sur le centre du village, où certains commencent à acquérir des terrains et des maisons. Alors que les estivants veillent à maintenir une forme de ségrégation entre l'habitat autochtone et le « quartier des villégiateurs » situé en bord de mer, les nouveaux arrivants sont des gentrifieurs, qui, loin d'opérer une éviction des couches populaires, vont chercher à en gouverner la diversité sociale (Tissot, 2011).

Dans les années 1980, la dissémination du nom de Pipa à travers le surf attire de nouveaux publics, moins intéressés par le surf que par la beauté des paysages, le cadre de vie et la culture locale (Loloum, 2017). Voyant là l'opportunité de vivre de leur passion, certains songent à s'installer définitivement à Pipa et démarrer une activité touristique (hôtel, restaurant, excursions, etc.). Il s'agit généralement d'anciens surfeurs ou de voyageurs de la première heure revenant sur leurs itinéraires vacanciers, mais cette foisci en couple (ou en famille) et avec les moyens de leurs ambitions. Issus d'un milieu aisé, le plus souvent diplômés du supérieur, ils disposent non seulement d'un capital financier, obtenu au cours de métiers antérieurs ou auprès de leur famille, mais aussi d'une « sensibilité culturelle » particulière, un style de vie « alternatif » et " raffiné », nourri par un environnement familial et l'imprégnation des modes naissantes du surf et des voyages.

Le tourisme et l'hôtellerie apparaissent ainsi comme une façon de valoriser ce style de vie de façon commerciale : 
Nombreux sont ceux qui deviennent hôteliers sans aucune formation, pour arrondir leurs fins de mois. (...) Ces petits hôteliers commercialisent un savoir-faire domestique jusqu'alors limité à un usage familial. Appartenant à la même classe moyenne urbaine que leurs clients, ils inventent une nouvelle formule d'accueil dont les services sont en adéquation avec leurs attentes. (Cousin et Réau, 2009, p. 56)

On retrouve ici l'hypothèse de reconversion sociale de l'avant-garde bourgeoise formulée par Bertrand Réau (2011) au sujet des inventeurs du Club Med et du tourisme d'aventure. En faisant la genèse sociale de ces nouvelles formules de vacances, Réau montre que leur succès repose essentiellement sur deux ressorts communs : premièrement la nécessité d'agents à fort capital de s'inventer de nouvelles voies d'autopromotion sociale lorsque les débouchés ordinaires sont saturés, et deuxièmement, l'homologie structurale entre les avant-gardes bourgeoises investissant leurs goûts pionniers dans l'offre touristique. Précurseurs dans l'exploration esthétique, ludique et morale du littoral brésilien, les «surfeurs »du littoral brésilien anticipent les aspirations d'une société travaillée en profondeur depuis le début du vingtième siècle par un imaginaire national en gestation depuis les années 1930 qui invite à la redécouverte d'espaces autrefois considérés comme marginaux, parmi lesquels le littoral et le Nordeste; par le passage d'une société agraire influencée par les modes européennes à une société urbaine et industrielle marquée par des valeurs individualistes et les influences nord-américaines; et enfin par une démocratisation des loisirs permise par la stabilité économique de l'aprèsguerre et le développement de l'industrie des voyages.

\section{De la distance comme ressource}

Dans les années 1980, le tourisme en est encore à ses premières heures. Les infrastructures sont précaires (Pipa n'obtient l'électricité et l'eau courante qu'en 1982), les lieux difficilement accessibles aussi bien par route que par voie aérienne, et la région reste peu connue à l'international. Ce n'est que dans les années 1990 que les pouvoirs publics investissent dans les infrastructures et la promotion, à travers le PRODETUR (Programme pour le Développement Touristique du Nordeste) soutenu par la Banque Internationale de Développement, permettant ainsi l'intégration de la région aux circuits internationaux de l'industrie touristique (Cruz, 2000). Aussi la plupart de ces jeunes entrepreneurs tardent à se lancer de plain-pied dans l'aventure du tourisme et de l'hôtellerie (les premiers hôtels n'ouvrent timidement qu'à la fin des années 1980). Contre l'idée d'une rupture radicale et soudaine, on constate que beaucoup entretiennent une relation distante avec la destination, profitant de quelques visites pour investir, mais sans pour autant s'installer. C'est notamment cette distance initiale, ce désintéressement relatif, qui leur permet de proposer une offre innovante tout en prenant le minimum de risques.

Le premier restaurant géré par l'un de ces surfeurs ouvre en 1984. Son propriétaire, Eduardo, ${ }^{7}$ est fils d'un officier de marine. Originaire de Rio de Janeiro, il vit depuis sa plus tendre enfance à Natal et fréquente Pipa depuis l'adolescence. Le « Rock Point » est d'abord conçu comme un établissement saisonnier, qu'il gère lors de ses séjours réguliers dans la station. Le reste du temps, il travaille comme ingénieur zootechnicien dans une grande entreprise d'élevage, dans le sud-est du pays. Comme beaucoup d'établissements ouverts par ces entrepreneurs de la dernière heure, le restaurant est administré en dilettante : 
Il n'y avait que deux plats au choix, raconte Eduardo : du poisson frit et du poulet rôti. On avait juste un frigo et une petite gazinière. Il ne nous en fallait pas beaucoup à l'époque. C'était le point de chute de tous les jeunes tarés qui passaient par là. Un autre surfeur a même ouvert un bar " self-service ». Une première dans l'histoire, je pense. Quand [le propriétaire] était fatigué, il laissait un frigo rempli de bières et il suffisait de laisser l'argent dans la tirelire. (Eduardo, 58, originaire de Rio de Janeiro, restaurateur. Entretien du 10 mai 2010, Pipa)

Beaucoup d'entreprises touristiques commencent ainsi comme des projets « pour les vacances ", jusqu'à ce que l'opportunité se présente de s'installer définitivement, une fois l'expérience acquise, les capitaux réunis et la famille constituée. C'est le cas de Victor, un publicitaire carioca arrivé pour la première fois en 1990 pour un séjour de surf, qui laisse son bar en gérance à un « local » avant de revenir 10 ans plus tard pour s'installer en tant qu'hôtelier.

J'ai tellement aimé l'endroit, la culture locale, que j'ai décidé d'acheter un petit lopin de terre avec l'idée de peut-être m'installer un jour. À l'époque personne n'achetait de «petits terrains ». Tout était grand, très grand. [Comment ça?] Parce que la terre ne valait rien. Pour qu'il y ait une plus-value, il fallait une certaine surface. Tu comprends? Sinon tu pouvais les échanger contre une télévision, un frigo, ça ne valait rien. [Mais un grand terrain, ça voulait dire combien?] Ça correspondait à ce terrain ici : 1,5 hectare, 2 hectares... (...) J'habitais à Rio. Un an après je suis revenu avec ma femme, je suis revenu pour voir la propriété, et comme je n'avais pas d'argent à l'époque pour faire une auberge, $\mathrm{j}$ 'ai ouvert un petit bar avec un ami autochtone : le Mirante da Pipa. On l'a ouvert avec lui et on l'a laissé en charge et on est retourné à Rio. C'était déjà lui qui s'occupait de la propriété en mon absence, je le payais pour qu'il surveille mon terrain. Ce n'est que 15 ans après que je suis venu m'installer et qu'on a démarré les bungalows. (Victor, 55, originaire de Rio de Janeiro, publicitaire et hôtelier. Entretien du 20 novembre 2013, Pipa)

Ces situations de gestion à distance donnent parfois lieu à des déconvenues, liées aussi au caractère « émergeant» de la destination, qui offre peu de sécurité institutionnelle aux investisseurs. L'un des voisins de Victor en fit un jour l'expérience : après avoir acheté une petite maison en bord de mer, il n'en retrouva à son retour l'année suivante que les fondations; on lui avait volé tous ses murs, brique par brique.

Cette gestion à distance permet également de faire connaître la station. Après la couverture médiatique offerte par les surfeurs, dont beaucoup disposent de contacts privilégiés avec les magazines spécialisés alors en pleine croissance, ce sont les premiers hôteliers qui prennent en charge la promotion touristique de Pipa. Tout d'abord de façon autonome en recevant dans leurs établissements des journalistes et autres " FamTours ${ }^{8}$ ", puis de façon organisée dans les salons internationaux à travers l'association des hôteliers qui voit le jour dans les années 1990. Les compétences acquises en amont enrichissent également l'activité touristique. Victor, le publicitaire carioca, contribue ainsi à la renommée de Pipa grâce à sa maîtrise précoce de l'Internet et des télécommunications. Il est le fondateur du premier site d'information touristique de la station, " Pipa.com.br ». À l'époque, son intérêt à faire connaître le lieu s'inscrit déjà dans un projet de valorisation immobilière et touristique :

J'ai créé le Portail Internet il y a 18 ans... Il n'y avait même pas de téléphone à l'époque, c'était de la folie. Personne ne savait ce que c'était Internet. J'ai dû faire une présentation publique pour expliquer ce que c'était. [Mais il y avait Internet quelque part ici?] Non, rien. Il $\mathrm{y}$ avait un mobile, mais qui captait très mal. Je me connectais au fournisseur de Rio et je transférais les données vers là-bas, octet par octet. (...) J'ai créé le site pour diffuser le lieu, parce que j'avais quelques terrains ici, pas vrai. Quand je suis revenu à Rio, j'ai laissé le site 
trois mois en ligne, et à mon retour les gens m'ont dit : « Dis donc, ça a bien marché ton truc, j'ai reçu plein de touristes. Il faut continuer ». Personne ne connaissait Pipa, c'était un coin paumé. (...) Ce site, pendant des années ça a été le principal véhicule de diffusion et de captation de touristes à Pipa. (Victor, entretien du 20 novembre 2013, Pipa)

\section{Investir davantage qu'un capital économique}

La décision d'ouvrir une auberge ou un hôtel dans une destination internationale tient à une combinaison de facteurs touchant à l'expérience des voyages, aux capitaux (culturels, économiques, sociaux) hérités de la famille, à la formation professionnelle et à l'étape du parcours familial. Pour beaucoup d'hôteliers interrogés, l'installation coïncide avec une mise en ménage et la parentalité. Pour d'autres, l'installation survient après une rupture personnelle (un nouveau départ après une séparation, une façon de commencer un projet commun avec un nouveau partenaire) ou professionnelle (une reconversion, un départ en retraite). L'opportunité d'une reconversion tient ainsi à la situation des individus par rapport à un cycle de vie. Bien que cohérent par rapport à un style de vie et à des parcours conventionnels bouchés, le choix du tourisme comme profession est bien souvent perçu comme un détour ou une bifurcation par rapport à un chemin déjà tracé. Il est logique par conséquent qu'il s'opère à l'occasion d'une rupture dans la trajectoire personnelle : positive lors de l'installation en famille, ou échappatoire lors d'une séparation ou d'un divorce.

Les premiers établissements touristiques ouverts par des outsiders sont de petites auberges aménagées dans des maisons achetées aux locaux à la fin des années 1980 . Certains sont tenus par des étrangers, pour qui le dollar confère un avantage de capital. C'est ainsi que naissent la Pousada dos Espanhois (l'Auberge des Espagnols) ou encore la Pousada da Aguias, appartenant à l'Italienne Silvia. Elle est l'une des premières étrangères à s'installer à Pipa, en 1985, après deux ans de voyage en Amérique Latine, et alors qu'elle découvre qu'elle est enceinte (« j'avais besoin d'un endroit calme pour élever mon enfant. J'étais baroudeuse à l'époque, il fallait que je me pose... »). Aujourd'hui, elle est propriétaire d'une auberge, d'une agence touristique, d'une galerie marchande et de plusieurs terrains en périphérie de Pipa.

Victor décrit ces premiers entrepreneurs étrangers comme des "rêveurs ", dont la motivation n'était pas tant d'investir que de trouver une « qualité de vie », à l'image de l'Allemand Depaches, un adepte du guru indien Osho aussi connu sous le nom de «Yahoo » :

Eux, ils voulaient surtout vivre ici, vivre, ce n'était pas des investisseurs. Je crois que le premier qui est venu investir avec un peu d'argent, c'est Depaches : "Yahoo ». Yahoo était un Allemand marié avec une mineira, Zora. Il avait habité à San Francisco et faisait partie de cette secte indienne : Osho. Il est venu avec sa femme et a acheté une maison en torchis dans la rue principale. Ils ont fait un restaurant et ils l'ont appelé « Yahoo », en hommage à leur secte. C'est à partir de là que les gens ont commencé à l'appeler Yahoo. Son restaurant était un restaurant de la qualité des restaurants des grandes villes... mais à Pipa. Donc il est venu avec de l'argent, comme investisseur, mais avec un style de vie, pas pour l'argent. Le restaurant c'était surtout pour se maintenir. (Victor, entretien du 20 novembre 2013, Pipa)

La relation entre entrepreneurs pionniers et population locale est souvent décrite sur le registre de l'amitié et du don de soi. L'Italienne Silvia insiste sur l'implication sociale et culturelle des nouveaux arrivants vis-à-vis de la communauté locale. Elle cite notamment 
Yahoo qui crée le premier Carnaval de Pipa en 1992 en finançant un bloco ${ }^{9}$ pour ses salariés autochtones, le Bloco de Yahoo, toujours en activité aujourd'hui :

\begin{abstract}
À l'époque il n'y avait pas de Carnaval à Pipa. Donc les serveurs de son restaurant voulaient tous aller faire la fête à Goianinha au lieu de travailler. Mais il les a convaincus de ne pas y aller en leur disant qu'en échange il organiserait un grand carnaval le jeudi, après le Mercredi des Cendres, quand les touristes seraient partis. Il leur a dit : " vous travaillez et après j'embauche un groupe de musique pour le carnaval et on fait notre fête. Je vous le promets! ». Et les gens : « mais tu vas vraiment le faire? » Et il l'a fait. C'est comme ça qu'il a créé le Bloco de Yahoo. Après il a fermé le restaurant, mais le Bloco est resté... (Silvia, 51, Italienne, investisseuse dans le tourisme et l'immobilier. Entretien du 28 octobre 2013, Pipa)
\end{abstract}

Luiz Francisco met également en avant son propre « engagement social » vis-à-vis de la population locale. Il propose des solutions innovantes pour tenter d'adapter sa gestion des ressources humaines à la main-d'œuvre locale qui est peu formée :

On travaille avec $85 \%$ de personnes locales et on essaye de se tenir à cet engagement. On a des formateurs et on leur donne une formation au premier emploi. [...] J'ai compris que les habitants d'ici donnent beaucoup d'importance à la vie personnelle. Du coup j'ai embauché plus de salariés, mais j'ai diminué leurs périodes de travail. Comme ça ils travaillent mieux et traitent mieux les clients. (Luiz Francisco, 52, originaire de l'État de São Paulo, hôtelier. Entretien du 6 août 2011, Pipa)

Le fait que les précurseurs touristiques ne soient pas mus dans un premier temps par la quête de profits économiques, mais plus par la recherche d'une " qualité de vie » et de profits symboliques, les encourage à inventer de nouvelles formules et à investir davantage qu'un simple capital financier. Beaucoup s'engagent dans les associations locales pour améliorer la qualité des infrastructures, de l'environnement et des services de la ville, d'autres font venir des organismes de formation pour offrir des cours à leurs employés. Il faut ainsi replacer la reconversion, qui est souvent présentée comme un processus individuel, dans un ensemble de trajectoires individuelles convergentes qui participent à valoriser la station touristique de façon globale.

Les premiers surfeurs et voyageurs ont d'une certaine manière créé leur propre offre touristique en inventant des nouvelles manières de voir et d'habiter le littoral. Forts d'un style de vie en vogue, à la fois subversif (par rapport au contexte politique et aux modes traditionnelles de villégiature) et dominant (car influencé par le modèle américain et diffusé par les milieux bourgeois et citadins), ils ont su faire de ce style un élément de distinction sociale et un objet d'attraction touristique : les premiers établissements ouverts par ces entrepreneurs pionniers étaient de fait des formes marchandes de cet art de vivre. Le fait de disposer d'un capital économique de départ ou d'un métier stable par ailleurs, ajouté au coût modéré des investissements fonciers sur cette partie inexplorée du littoral, offre une sécurité propice à des reconversions « audacieuses » car peu inquiètes de la rentabilité immédiate du projet : «La sécurité que procure la certitude intime de pouvoir compter sur une série de "filets de protection" est au principe de toutes les audaces, y compris intellectuelles, que leur insécurité anxieuse de sécurité interdit aux petits-bourgeois » (Bourdieu, 1974, p. 14).

\title{
De la plantation à l'hôtel : les reconversions touristiques de l'élite agraire
}

Outre les anciens surfeurs, on retrouve parmi les entrepreneurs touristiques pionniers quelques individus issus des familles de grands propriétaires terriens, d'éleveurs ou de planteurs de canne à sucre de la région. Alors qu'ils ont été formés initialement en agronomie, le métier 
d'hôtelier est perçu comme une rupture dans leur trajectoire professionnelle. D'une certaine manière, la transition de l'agronomie à l'hôtellerie traduit la crise de modernisation qui affecte le milieu agricole au cours de la deuxième moitié du vingtième siècle, et en particulier le secteur sucrier, qui voit disparaitre les moulins à canne traditionnels (engenhos) au profit de grandes distilleries agro-industrielles (usinas) destinées à la production d'éthanol. Cette situation a pour effet de réduire considérablement les débouchés agricoles pour les fils de l'élite rurale, contraints de se reconvertir (Garcia, 1993b). Dans le même temps, le tourisme et l'immobilier offrent de bonnes possibilités de conversion pour l'élite agraire, forte d'un patrimoine foncier, habituée à valoriser économiquement la terre et le paysage, mais aussi porteuse d'une légitimité sociale et culturelle. Cela explique pourquoi on retrouve beaucoup de descendants de propriétaires ou cadres de plantations sucrières parmi les hôteliers de Pipa, une station située à l'orée d'une importante zone de plantations.

\section{Les usages légitimateurs du voyage}

Le goût pour les voyages est souvent avancé comme un argument légitimant une vocation hôtelière. C'est le cas par exemple de Jorgio, propriétaire d'un charmant hôtel en bord de mer, qui nous reçoit dans une véranda au sol en béton ciré et aux meubles en bois patiné. Lorsqu'on l'interroge sur les raisons qui l'ont poussé à s'installer à Pipa, il répond que ce sont les voyages qui ont déclenché chez lui ce choix de l'hôtellerie. C'est dans les années 1980, après avoir séjourné dans plusieurs destinations côtières au Brésil et à l'étranger, que lui vient l'idée de démarrer un hôtel ici, alors que Pipa n'est encore qu'une toute petite destination émergente :

Les voyages t'apportent une sensibilité particulière, c'est en étant toi-même un voyageur que tu apprends à répondre aux attentes de tes clients. Ça ne s'apprend pas à l'école. (...) Quand je me suis marié, en 1985, j'ai commencé à voyager avec mon épouse. On a connu Porto Seguro, Ilheus, qui étaient des stations jeunes au Brésil... C'était encore le début de la plage dans le Nordeste et je me suis dit que ça pouvait marcher ici. (Jorgio, 57, originaire de Goianinha, hôtelier. Entretien du 12 septembre 2013, Pipa)

Mais en s'intéressant plus attentivement à son parcours, on comprend que beaucoup d'autres facteurs rendent possible la réalisation de cette "vocation ». Face à " l'illusion biographique » (Bourdieu, 1986) faisant de l'enchantement des voyages un déclencheur miraculeux de nouvelles vocations, il est important de décrypter les possibilités objectives de ces reconversions.

Quand Jorgio achète une parcelle de plus de 230 hectares pour y planter de la canne, en 1982, le fait que son terrain dispose d'un accès privilégié à la mer ne le concerne pas plus que ça; ce n'est qu'après son expérience des voyages et l'ouverture des premiers établissements dans le voisinage qu'il décide de se lancer dans l'hôtellerie. Rien ne semblait a priori destiner Jorgio aux métiers du tourisme : fils d'un planteur de canne à sucre de la région, il a fait des études d'agronomie après s'être essayé dès l'âge de 18 ans à l'agriculture sur une parcelle donnée par son père. Il a pourtant de par son appartenance à l'aristocratie sucrière locale toutes les prédispositions pour accéder au marché touristique. Sa femme, elle aussi issue d'une famille de notables de la région, n'est autre que la notaire de la commune voisine, une fonction héritée de son père. De même, le déclin des plantations traditionnelles et la modernisation de l'agriculture encouragent les conversions en limitant les opportunités pour les fils de planteurs et complexifiant le travail de gestion des nouveaux agriculteurs, obligés de recourir aux démarches administratives pour accéder aux programmes du gouvernement. ${ }^{10}$ 
On le comprend ici, ce ne sont pas seulement les voyages, comme il le laisse entendre, qui lui ont permis d'entrer dans cette vocation hôtelière, mais aussi un ensemble de conditions sociales, économiques et culturelles. Les ressources acquises dans l'expérience de la mobilité suffisent rarement à expliquer le succès d'une trajectoire : le capital international est toujours « combiné » avec d'autres formes de capitaux (Wagner et Réau, 2015). Dans le cas des hôteliers issus de l'élite agraire, c'est la combinaison entre un capital international, un ancrage territorial et un statut social dominant qui confère une position privilégiée dans le champ touristique.

Comme le souligne Anne-Catherine Wagner (2007), l'accès au voyage est surtout un avantage symbolique et moral par rapport à ceux confinés à la sédentarité et au « local ». La mobilité n'a évidemment pas la même signification en fonction des milieux sociaux, des contextes nationaux et des milieux professionnels. Alors que pour les milieux populaires et les populations immigrées, la mobilité est le plus souvent contrainte et synonyme de stigmate, dans les milieux favorisés elle constitue une norme voire une marque de statut.

Dans le domaine du tourisme et de l'hôtellerie, le capital international est à la fois ressource et contrainte. Il faut en effet « connaître la clientèle », comme me l'a signifié Jorgio. Or quoi de plus logique pour comprendre les touristes que d'en être un (ou d'en avoir été un) soi-même, de parler leur langue, de partager leurs aspirations, autrement dit de disposer en tant qu'entrepreneur d'un « capital cosmopolite » (Weenink, 2007), que ce soit sous une forme incorporée (un savoir-être international), institutionnalisée (des diplômes internationaux) ou objectivée (avoir des employés européens, des meubles importés, des filiales à l'étranger). Mais les métiers de l'accueil exigent également l'exercice d'une discipline et d'une certaine forme de sédentarité. C'est typiquement ce mélange de culture internationale et de tradition de métier qui est enseigné dans les écoles d'hôtellerie, toujours en recherche de candidats et d'enseignants ayant le sens de l'international, mais restant attachés à des coutumes nationales et à des codes professionnels stricts.

\section{Le rendement touristique des sports d'élite}

Les ressources sociales et culturelles acquises à travers la pratique de certains loisirs peuvent être réinvesties ailleurs et reconverties en ressources touristiques. Cela vaut aussi bien pour les pratiques émergentes à fort rendement symbolique, comme le surf, que pour les sports d'autres sports d'élite comme la natation (Réau, 2007), la voile et les sports équestres (Lucena, 2000).

Romualdo est un autre exemple de réinvestissement des ressources traditionnelles de l'élite agraire dans le tourisme. Propriétaire d'un hôtel de bord de mer depuis 1992, il a été l'un des premiers hôteliers à s'installer dans la région, en même temps que quelques surfeurs et expatriés. Âgé d'une cinquantaine d'années, il est le descendant d'une importante famille de notables et d'anciens maîtres de plantation. Son accès privilégié au marché foncier local (de par ses réseaux familiaux et son nom) lui a permis d'acquérir non seulement un terrain en bord de mer, espace traditionnellement investi par les estivants depuis le début du vingtième siècle, mais aussi un ranch de plusieurs dizaines d'hectares à quelques kilomètres de Pipa, dans une zone de latifundia encore occupée par la canne à sucre et l'élevage, et qui constitue aujourd'hui un nouveau front d'expansion pour la ville touristique.

Comme beaucoup d'autres hôteliers issus de l'élite agraire, il s'est formé en agronomie et a gardé de son héritage familial un profond attachement à la terre. Il manifeste cet attachement à travers un goût prononcé pour les chevaux et les 
vaquejadas, sorte de corridas brésiliennes pratiquées à cheval et consistante à capturer une vache en la tirant par la queue. Face à l'impossibilité de combiner ce sport - qui a lieu les week-ends - avec son activité hôtelière, il se reporte sur les randonnées équestres, qu'il vend sous forme d'excursions aux clients de son hôtel. Dans les années 1990, il aménage un ranch à la périphérie de Pipa dans une propriété achetée à un parent. En 2000, alors qu'il possède plus de 25 chevaux, il décide de se lancer dans l'élevage : «\$146\#plus par passion que par nécessité », précise-t-il. Il transforme peu à peu son ranch en haras spécialisé dans le Mangalarga Marchador, une race de trotteurs typiquement brésilienne et particulièrement adaptée à la randonnée. Depuis, Romualdo partage son temps entre les concours équestres, les randonnées à cheval et son hôtel. Il est aujourd'hui l'un des principaux entrepreneurs de Pipa et un éleveur de chevaux reconnu.

La trajectoire de Romualdo illustre comment un capital culturel porté à travers les sports équestres symboles de l'héritage colonial et du pouvoir des barons peut être reconverti dans le tourisme. Dans un autre registre, les vaquejadas peuvent également rapporter gros : traditionnellement un rite de prestige rappelant le travail de conduite du bétail dans les campagnes nordestines pratiqué par les éleveurs lettrés (Cascudo, 1976), les vaquejadas contemporaines font désormais l'objet dans d'un véritable négoce culturel, avec ses professionnels, ses investisseurs, ses émissions de télévision et ses entreprises spécialisées (Barbosa, 2006).

\section{Commercialiser un style de vie}

Parce que leur reconversion relève autant du choix professionnel que du choix de vie, ces jeunes entrepreneurs ouvrent des établissements qui leur ressemblent. En investissant dans leur auberge comme ils investiraient dans leur maison, ils plaisent aux voyageurs qui partagent avec eux les mêmes goûts et ambitions esthétiques.

Le cas de Luiz Francisco illustre bien ce principe d'adéquation sociologique, d'homologie structurale, entre l'offre d'hébergement et la demande touristique naissante. Luiz Francisco est le propriétaire d'un ecoresort de luxe (entre 250 et 450 US\$ la nuit) régulièrement cité dans les magazines parmi les " itinéraires de charme » de la région. L'entrée de l'établissement est signalée par de larges massifs verdoyants mimant l'aspect d'une forêt primaire. À l'intérieur, les bungalows sont entourés de massifs méticuleusement jardinés pour leur donner une allure luxuriante et sauvage. Les bâtiments sont reliés entre eux par des petits chemins en galets recouverts de mousse. Dans les chambres, une literie fine, des sculptures d'art, un mobilier soigné et une robinetterie dorée. Parmi les plus luxueux de la destination, son hôtel a pourtant commencé comme une simple auberge.

Le parcours de Luiz Francisco est emblématique du processus de reconversion qui caractérise l'arrivée des premiers hôteliers dans le Nordeste. Originaire de l'État de São Paulo, il s'est lui aussi formé en agronomie à São José do Rio Preto, une formation courante parmi les fils de l'élite traditionnelle attachée à la sauvegarde de son patrimoine agraire et foncier. Luiz Francisco a travaillé pendant cinq ans en tant que gérant d'une usine à sucre, où il dit avoir acquis ses compétences de " gestionnaire et d'organisateur ». Mais ce métier n'était manifestement pas pour lui, ayant accepté ce poste « surtout pour faire plaisir à [s]on père », qui y travaillait en tant que dentiste. Il était alors déjà sensible à l'agriculture biologique et avait effectué plusieurs séjours dans des " communautés hippies ", avant de se rendre à l'évidence qu'il était " trop chef » pour ce genre d'entreprises humaines. Un jour, alors que sa femme est enceinte de leur premier enfant, il démissionne de l'usine pour ouvrir une épicerie fine à São 
Paulo, au grand dam de ses parents et beaux-parents qui voyaient en lui un futur « capitaine d'industrie ». Peu après la naissance de leur fille, au milieu des années 1980, il vend l'épicerie à son beau-frère et déménage à Pipa, dont il avait entendu parler après avoir voyagé à Trancoso et Arraia d'Ajuda, deux stations balnéaires montantes de l'État de Bahia. Il acquiert rapidement un terrain et souligne le côté « aventureux » de l'entreprise :

Le terrain était situé entre la décharge et le cimetière. Imagine, faire du tourisme dans une situation pareille! Mais j'ai pensé : « s'il n'y a que ce terrain qu'on peut acheter, alors allonsy! » On a vendu l'épicerie et on est venu ici. Moi, mon ex-femme et ma fille... (Luiz Francisco, 52, originaire de l'État de São Paulo, hôtelier. Entretien du 26 août 2011, Pipa)

Il est intéressant de noter l'influence de l'entourage familial dans le parcours de Luiz Francisco, qui, bien qu'opposé sur le principe à une telle rupture, fournit les ressources nécessaires à sa reconversion : tout d'abord le père en lui facilitant l'entrée dans l'usine, ensuite le beau-frère en rachetant son épicerie. L'origine sociale favorisée de Luiz Francisco et de sa femme semble leur conférer un goût « inné » pour l'accueil. Luiz Francisco décrit ainsi le style des premiers bungalows qu'il met en location pour les touristes :

Tout était très simple, mais bien arrangé et stylé. C'est comme ça que tout d'un coup, je me suis retrouvé dans l'hôtellerie. On n'avait pas grand-chose, mais on savait faire plaisir aux clients. On faisait tout avec amour et ça a marché. On a commencé à acheter d'autres terrains autour et le succès est arrivé. (Luiz Francisco, entretien du 26 août 2011, Pipa)

L'hôtellerie leur permet ainsi de convertir un goût raffiné pour la décoration intérieure en un service commercial. Le cas de Luis Henrique rappelle l'exemple des inventeurs du Club Med analysé par le sociologue Bertrand Réau (2007), qui montre comment l'investissement dans de nouvelles formules de loisirs a permis à de jeunes aristocrates confrontés à des débouchés bloqués et à un risque de déclassement de se promouvoir socialement en convertissant un capital social et culturel (acquis au sein de clubs de natation réservés à l'aristocratie) en capital économique.

Comme c'est souvent le cas dans les récits fondateurs, l'insistance sur les improvisations du début cache en réalité un travail méticuleux de mobilisation de ressources et de réseaux de relations préexistants. Le détachement initial vis-à-vis des nécessités matérielles de l'existence la « vie de bohème » sur laquelle s'attardent les entrepreneurs pionniers dans leur récit - montre que leur activité est considérée comme " un état, une manière d'être plus que comme une profession » (Saint Martin, 2000, p. 791).

Comme pour les clubs de vacances, l'hôtellerie distinctive et « écologique » telle que celle proposée par Luiz Francisco apparaît comme une manière de « commercialiser avec succès leur mode de vie. En "avance" sur les goûts de leur classe sociale, l'investissement de leur "sensibilité culturelle" dans l'espace des loisirs et du tourisme apparaît comme un moyen de se reclasser » (Réau, 2011, p. 120).

L'innovation découle ainsi d'une adéquation sociologique - d'un « bricolage réussi » (Réau, 2007, p. 68) - entre l'hôtelier et son public. La segmentation de l'offre, partagée entre, d'une part, d'anciens surfeurs ou hippies friands de formules d'hébergement et restauration « rustiques », et d'autre part, des entrepreneurs au style plus sophistiqué issus de l'élite agraire, appelle à son tour une segmentation de la demande : un bar " self-service » et un hôtel de charme n'attirent pas la même clientèle. Cette adéquation sociologique entre offre et demande à partir d'une convergence des goûts et des habitus tend à remettre en cause la théorie néo- 
classique du marché qui voudrait que l'offre (les producteurs) et la demande (les touristes) se rencontrent abstraitement sur un marché, sans médiations sociales.

\section{Conclusion}

Comment la pratique des loisirs et des voyages peut-elle favoriser les reconversions professionnelles? Comment parvient-on à « changer de cap » pour s'investir dans des métiers proches de ses passions ludiques ou de ses destinations de voyage?

Les injonctions hédonistes incitant tout un chacun à se libérer du " train-train » quotidien pour adopter des modes de vie et des métiers plus en accord avec ses plaisirs éludent bien souvent les contraintes et conditions particulières d'une telle rupture. Le choix du tourisme comme activité professionnelle apparait comme un moyen de concilier l'astreinte du travail avec l'univers des loisirs ou des voyages. Il parait toutefois illusoire de présenter ces inflexions biographiques comme des « bifurcations », c'est-à-dire comme des ruptures complètes avec la séquence précédente. Il s'agit le plus souvent de « reconversions » personnelles et professionnelles qui consistent à transposer dans un nouveau domaine d'activité des ressources acquises par ailleurs, non seulement dans les loisirs et les voyages, mais aussi dans la famille, la formation, le réseau social, etc. De nombreux héritages sont mobilisés. Et même si les secteurs ici en question (l'hôtellerie, la restauration, l'accueil) ne nécessitent pas forcément de grands diplômes, ils requièrent néanmoins des ressources spécifiques (un savoir-être, des savoir-faire relationnels, des dispositions au leadership) qui sont bien souvent acquises en amont.

Comme le montre le cas des entrepreneurs de Pipa, l'affichage ostentatoire des héritages du surf et des voyages dissimule souvent une origine sociale et des parcours professionnels offrant des prédispositions propices à l'élection du tourisme comme métier. Bien loin de l'éthique de détachement matériel, voire de dénuement des surfeurs et voyageurs hippies des années 1970, le parcours des hôteliers et restaurateurs rencontrés montre qu'ils disposaient d'un capital économique confortable, un accès privilégié au foncier et au crédit, un bon niveau de formation et des goûts culturels dominants.

Même lorsqu'ils n'avaient de "surfeurs » que le nom ou le style vestimentaire, ces jeunes bourgeois charriaient avec eux tout un univers culturel et social qui parlait aux touristes « à l'âme en secret ». L'attachement à des univers sportifs, ludiques ou artistiques socialement valorisants (comme le surf ou l'équitation) adossés à des modèles culturels d'avant-garde et dominants (comme la contre-culture américaine ou l'univers équestre) accroît en effet les chances de réussite de reconversion professionnelle dans le tourisme. Ces activités rencontrent plus facilement un écho favorable parmi des consommateurs désireux d'accéder à ces styles de vie distinctifs. L'engagement dans des pratiques émergentes offre un espace des possibles varié pour s'y investir économiquement et socialement, à la fois parce que les coûts d'accès sont généralement moins élevés par rapport à un marché consolidé, mais aussi parce que le caractère minoritaire et distinctif de la pratique augmente les profits symboliques.

Même lorsque l'investissement de départ est modéré, la création d'une nouvelle entreprise contient nécessairement une part de risque. C'est pourquoi beaucoup d'entrepreneurs touristiques s'y essayent d'abord « en dilettante », par petites touches et à distance, en préservant un emploi régulier. Cela leur laisse le temps d'accumuler les ressources nécessaires pour se donner les moyens de leurs ambitions. Garder une attache professionnelle ou résidentielle dans une grande ville (comme beaucoup l'ont fait au début) permet également de se garder une échappatoire en cas d'échec : une sécurité au principe des reconversions les plus osées. Cette observation contredit l'idée d'une 
reconversion envisagée comme une « rupture » biographique totale. Il s'agit en réalité d'un processus itératif, opérant par tâtonnement et par opportunités.

Ces reconversions surviennent par ailleurs à des moments particuliers des cycles de vie. De la même manière qu'on ne part pas facilement pour un tour du monde au moment de fonder famille, la reconversion en tant qu'hôtelier ou restaurateur dans une destination tropicale coïncide bien souvent avec un changement dans le parcours personnel ou familial : l'envie de fonder famille, de dépasser une séparation, de réinvestir une indemnité de licenciement, etc.

Le cas des entrepreneurs interrogés dans cette étude est utile pour penser les reconversions professionnelles dans les métiers du tourisme. La passion des voyages ne suffit pas elle seule à expliquer les possibilités de reconversion professionnelle. Il faut considérer les prédispositions économiques, sociales et culturelles nécessaires à l'accomplissement d'une carrière dans le tourisme, ainsi que l'inscription de la reconversion dans le cycle de vie. Il est par ailleurs plus avantageux de se reconvertir dans des lieux ou des métiers associés à des pratiques émergentes (comme le surf dans les années 1970), pour lesquelles l'investissement initial est modéré et les rendements (symboliques et commerciaux) sont maximums. L'adéquation sociologique entre touristes et promoteurs du tourisme est un autre facteur de succès des reconversions. La particularité de l'hôtellerie et de la restauration est de commercialiser des pratiques habituellement limitées à l'usage domestique et familial. C'est l'homologie de classe entre touristes et hôteliers/restaurateurs qui permet d'expliquer que les uns trouvent spontanément chez les autres ce qu'ils sont venus chercher. Les deux se rejoignent intimement dans leurs goûts et dans leurs aspirations.

$\mathrm{Au}$ terme de cette analyse, on comprend l'intérêt de questionner les injonctions contemporaines invitant à « tout plaquer » pour gagner sa vie en voyageant et travailler en s'amusant. Au-delà des illusions biographiques faisant de la passion, du courage et de l'esprit de subversion comme moteur de la rupture, on a vu avec les entrepreneurs de Pipa que de telles reconversions requièrent une variété de conditions et de ressources. Il serait intéressant dans de futures recherches d'étendre ce questionnement à des professions plus actuelles, à l'instar des «digital nomads », qui prétendent faire de la confusion entre loisir/travail ou voyage/travail une nouvelle norme, en omettant bien souvent de mentionner les coûts spécifiques et ressources nécessaires à l'adoption de tels styles de vie.

\section{Déclaration}

Aucun conflit d'intérêts potentiel n'a été rapporté par l'auteur.

\section{Notes}

1. «Get-up-and-go generation », c'est ainsi qu'est sous-titré le magazine easyJet Traveller, disponible à bord des avions de la compagnie low-cost du même nom.

2. Espaces intégrant hébergement, restauration et travail proposés par de grandes entreprises de services comme Google ou AirBnB concevant l'espace de travail comme un coffee shop ou par des sociétés indépendantes au nom évocateur («Surf Office ", "Cowocamps ", « Easywork », « Néo-Nomade »), louant des espaces de " co-working » spécialement aménagés pour le télétravail. À ce sujet, voir le dossier spécial paru dans la revue Espaces, « Travail et vacances. Nouveaux rythmes, nouveaux lieux », $\mathrm{n}^{\circ}$ 330, mai-juin 2016.

3. Évoquons par exemple l'entreprise Remote Year, qui offre la possibilité à des «digital nomads » ou « remote workers » de passer un an à « travailler en voyageant et en explorant douze villes autour du monde pendant un an »- pour la bagatelle de 2000 dollars par mois (http://www. remoteyear.com). 
4. Définie comme un « système de dispositions durables et transposables, structures structurées prédisposées à fonctionner comme structures structurantes, c'est-à-dire en tant que principes générateurs et organisateurs de pratiques et de représentations qui peuvent être objectivement adaptées à leur but sans supposer la visée consciente des fins et la maîtrise expresse des opérations nécessaires pour les atteindre » (Bourdieu, 1980, p. 88).

5. La crise de 1929, la centralisation progressive du pouvoir et un héritage des sports aquatiques donnant priorité aux courses de voiliers et à l'aviron comme pratique distinctive sont les principaux facteurs qui expliquent que le surf ne se soit pas développé plus tôt en tant que subculture parmi la jeunesse brésilienne du début du vingtième siècle.

6. À quelques kilomètres de Pipa, Natal la capitale du Rio Grande do Norte accueil l'une des principales bases aériennes américaines (connu comme le " Trampoline de la Victoire ») ayant pour objectif le contrôle de l'espace atlantique.

7. Les noms des enquêtés ont été changés pour respecter leur anonymat.

8. Les Familiarization Tours sont des séjours organisés à destination des professionnels du tourisme (agences de voyages, tour-opérateurs, médias spécialisés) et visant à leur faire connaître les différents produits et atouts touristiques d'une destination. Pour les hôteliers impliqués dans la réception de ces FamTours, l'enjeu est bien évidemment de faire la publicité de leur établissement, mais aussi de la destination en général.

9. Un bloco pendant le Carnaval est un rassemblement semi-organisé de personnes décidant de défiler ensemble.

10. C'est le cas de Rodrigo, qui bénéficia du plan Pro-Alcool, un programme mis en œuvre en 1975, après le premier choc pétrolier, pour encourager la production d'éthanol (à partir de canne à sucre). C'est notamment grâce à ce plan qui prévoyait un accès facilité à l'emprunt, à la mécanisation et aux produits phytosanitaires, que Rodrigo a pu acheter le terrain agricole sur lequel il a par la suite établi son hôtel.

\section{Références}

Abbott, A. (2001). Time matters. On theory and method. Chicago: University of Chicago Press.

Arias, M. (2002). Surf Gênese. A história da evolução do surf. São Paulo: Alma Surf.

Barbosa, E. L. (2006). Valeu boi! O negócio da vaquejada. Teresina: EDUFPI.

Bertaux, D. (1980). L'approche biographique : sa validité méthodologique, ses potentialités. Cahiers Internationaux de Sociologie, 69, 197-225.

Bertho Lavenir, C. (1999). La Roue et le stylo. Comment nous sommes devenus touristes? Paris : Odile Jacob.

Bourdieu, P. (1974). Avenir de classe et causalité du probable. Revue Française de Sociologie, 15, 3-42. doi :10.2307/3320261.

Bourdieu, P. (1979). La distinction. Critique sociale du jugement. Paris : Minuit.

Bourdieu, P. (1980). Le sens pratique. Paris : Minuit.

Bourdieu, P. (1986). L'illusion biographique. Actes de la recherche en sciences sociales, 62/63, 6972. doi :10.3406/arss.1986.2317.

Cascudo, L. C. (1976). A vaquejada nordestina e sua origem. Natal: Fundação José Augusto.

Cousin, S., et Réau, B. (2009). Sociologie du tourisme. Paris : La Découverte.

Cruz, R. C. (2000). Política de turismo e território. São Paulo: Contexto.

Dantas, E. (2009). Maritimidade nos trópicos: por uma geografia do litoral. Fortaleza: Edições UFC.

Defrance, J. (1989). Un schisme sportif. Clivages structurels, scissions et oppositions dans les sports athlétiques, 1960-1980. Actes de la recherche en sciences sociales, 79, 76-91. doi :10.3406/ arss.1989.2908.

Dobry, M. (2010). Le politique dans ses états critiques : retour sur quelques aspects de l'hypothèse de continuité. Dans M. Bessin, C. Bidard, et M. Grossetti (dirs.), Bifurcation, les sciences sociales face aux ruptures et à l'événement (pp. 64-88). Paris : La Découverte.

Dumazedier, J. (1962). Vers une civilisation des loisirs? Paris : Le Seuil.

Garcia, A. (1993a). Les intellectuels et la conscience nationale au Brésil. Actes de la recherche en sciences sociales, 98, 20-33. doi :10.3406/arss.1993.3049.

Garcia, A. (1993b). Reconversion des élites agraires : du pouvoir local au pouvoir national. Études rurales, 131/132, 89-105. doi :10.3406/rural.1993.3433. 
Grossetti, M. (2010). Imprévisibilités et irréversibilités : les composantes des bifurcations. Dans M. Bessin, C. Bidard, et M. Grossetti (dirs.), Bifurcation, les sciences sociales face aux ruptures et à l'événement (pp. 147-159). Paris : La Découverte.

Guibert, C. (2007). Le premier âge du surf en France : un sport socialement sélectif. Science \& Motricité, 61, 89-100. doi :10.3917/sm.061.0089.

Guibert, C. (2011). Surf et « contre-culture » : la dimension symbolique des constructions journalistiques de la presse spécialisée en France. Sciences sociales et sport, 4, 11-39. doi :10.3917/ rsss.004.0011.

Guillaume, C., et Pochic, S. (2010). Mobilité internationale et carrières des cadres : figure imposée ou pari risqué? Formation emploi, 112, 39. doi :10.3917/form.112.0039.

Hughes, E. (1996). Le regard sociologique. Essais choisis. Paris : Éditions de l'EHESS.

Jaxel-Truer, P. (2016,7 août). Au Brésil, comment Trancoso est devenu le rendez-vous de la jetset. Le Monde.

Lafortune, J.-M. (2007). Les règles de l'ostentation : l'œuvre phare de Veblen : source et guide de la sociologie du loisir. Revue Interventions économiques, 36. Récupéré de http://interventionseco nomiques.revues.org/537

Loloum, T. (2015a). Derrière la plage, les plantations. Ethnographie d'une situation touristique dans le Nordeste brésilien (Thèse d'anthropologie sociale et études du tourisme). Écoles des Hautes Études en Sciences Sociales - Université de Lausanne.

Loloum, T. (2015b). La situation touristique. Reconfigurations sociales dans une station balnéaire brésilienne. Tsantsa, 20, 163-167.

Loloum, T. (2017). Derrière la plage, les plantations: touristification du littoral et recomposition des élites dans le nordeste brésilien. Actes De La Recherche En Sciences Sociales, 218, 46-63. doi: $10.3917 /$ arss.218.0046.

Lucena, R. F. (2000). Esporte na cidade. Aspectos do esforço civilizador brasileiro. São Paulo: Editora Autores Associados.

Napolitano, M. (2002). Cultura e poder no Brasil contemporâneo. Curitiba: Juruá Editora.

Oliveira, H. (2015, 20 mars). Uma onda sem fim. Jornal Zona Sul.

Patha, C. (2016). Roaming: Living and working abroad in the 21st century. London: Either/Or Press.

Perrier, M. (2015). «Wwoofer » en Nouvelle-Zélande, entre tourisme et travail. Pour, 227, 31-39. doi :10.3917/pour.227.0031.

Réau, B. (2007). S'inventer un autre monde. Le Club Méditerranée et la genèse des clubs de vacances en France (1930-1950). Actes de la recherche en sciences sociales, 170, 66-87. doi : $10.3917 /$ arss. 170.0066 .

Réau, B. (2011). Les Français et les vacances. Paris : CNRS Éditions.

Rivron, V. (2007). Le reclassement de la musique populaire brésilienne - trajectoires de producteurs radiophoniques et construction d'un patrimoine national (1936-1970). Regards sociologiques, 33/34, 55-67.

Saint Martin, M. (2000). Vers une sociologie des aristocrates de classes. Cahiers d'histoire, 45(4), $785-801$.

Tissot, S. (2011). De bons voisins. Enquête dans un quartier de la bourgeoisie progressiste. Paris : Raisons d'agir.

Veblen, T. (1899). Théorie de la classe de loisir. Paris : Gallimard, édition 1970.

Viry, G., Kaufmann, V., et Widmer, E. (2009). La grande mobilité géographique pour des raisons professionnelles en Suisse : une étape de vie préparentale? Recherches familiales, 6, 67-80. doi : $10.3917 /$ rf.006.0067.

Wagner, A.-C. (2007). La place du voyage dans la formation des élites. Actes de la recherche en sciences sociales, 170, 58-65. doi :10.3917/arss.170.0058.

Wagner, A.-C., et Réau, B. (2015). Le capital international : un outil d'analyse de la reconfiguration des rapports de domination. Dans J. Siméant (dir.), Guide de l'enquête globale en sciences sociales (pp. 33-46). Paris : CNRS Éditions.

Weenink, D. (2007). Cosmopolitan and established resources of power in the education arena. International Sociology, 22(4), 492-516. doi:10.1177/0268580907078010. 